\title{
Nueva plataforma dinamométrica para el análisis y valoración del rendimiento deportivo
}

\author{
SANCHEZ-LACUESTA, J., COMIN, M., PRAT, J., SOLER, C., DEJOZ, R., PERIS, J.L., HOYOS, J.V., VERA, P.
}

Instituto de Biomecánica de Valencia, Valencia (Spain)

\section{RESUMEN}

Un nuevo modelo de plataforma dinamométrica ha sido desarrollado por el Instituto de Biomecánica de Valencia (IBV) y se han fabricado sus primeros prototipos. Permite la medida de la fuerza de reaccipón tridimensional, en momento torsor y el punto de aplicación de la fuerza, a una velocidad de muestreo de hasta 1000 $\mathrm{Hz}$ ( $500 \mathrm{~Hz}$ para dos plataformas).

El nuevo diseño se caracteriza por poseer cuatro captadores bidimensionales octogonales mecánicamente desacoplados, placa superior de rigidez optimizada y sensibilidad cruzada mínima.

Las plataformas dinamométricas son instrumentos electrónicos para la medida y análisis de la fuerza que un individuo ejerce sobre el suelo.

Su utilización masiva como herramienta fundamental por parte de grupos de investigación en Biomecánica se ha producido a lo largo de los últimos veinte años destacando, entre sus múltiples aplicaciones, el análisis y valoración de la técnica de deportistas así como el diagnóstico y evaluación de tratamientos de patologías del aparato locomotor.

El Instituto de Biomecánica de Valencia ha desarrollado, en los últimos años, diversas generaciones de plataformas dinamométricas. A finales de 1990 se abordó el diseño de un nuevo prototipo industrializable que mejorase los existentes.

La plataforma diseñada se basa, como las anteriores, en el empleo de transductores extensométricos, cuyo buen comportamiento a frecuencias bajas y alta linealidad los hace particularmente indicados para el estudio de movimientos humanos.

La nueva plataforma se instrumenta mediante cuatro captadores bidimensionales octogonales, a cada uno de los cuales se han adherido ocho galgas extensométricas. Con ellas se configuran dos puentes de Wheatstone, uno de ellos sensible a acciones verticales y el otro a acciones transversales y ambos compensados térmicamente.

La disposición de las galgas en el octógono obedece a estudios realizados mediante modelado por Elementos Finitos y anula, teóricamente, las interferencias cruzadas entre ambas direcciones de sensibilidad.
El diseño mecánico del captador se ha perfeccionado en esta ocasión con objeto de aislarlo mecánicasmente frente a esfuerzos en la tercera dirección así como frente a momentos flectores y torsores. Para ello se han introducido tres grados de libertad adicionales en cada captador combinando una rótula esférica y un par de rotación.

El nuevo diseño simplifica, además, el proceso de calibración y puesta a punto de la plataforma. Los captadores se numeran y calibran independientemente, antes de su montaje sobre la placa, y sus coeficientes de calibración quedan registrados en un fichero de configuración para su utilización posterior.

Los cuatro captadores se disponen en las cuatro esquinas de la plataforma (figura 1). Los sensores 1 y 4 son senbibles a fuerzas verticales y antero-posteriores, mientras que los sensores 2 y 3 permiten medir fuerzas verticales y medio-laterales. De este modo es posible medir la fuerza resultante en las tres direcciones del espacio así como calcular el momento torsor y el centro de presiones verticales, como se expone más adelante.

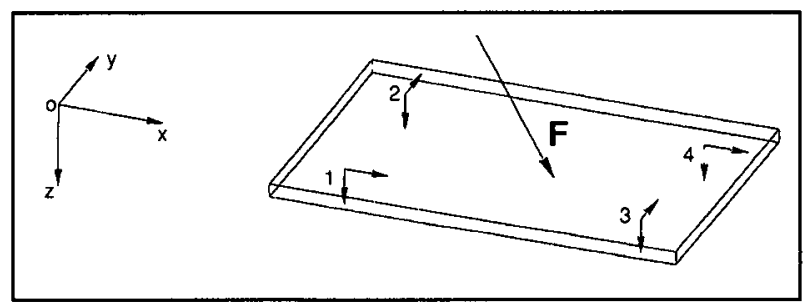

Fig. 1. Posición de los captadores sobre la plataforma.

La placa superior del instrumento ha sido, a su vez, rediseñada. Consiste en una plancha gruesa de aluminio macizo cuya cara inferior ha sido vaciada de material. A partir de un estudio teórico de la distribución de tensiones sobre la placa se han aligerado más las zonas con menor solicitación de esfuerzos. De este modo se conserva la rigidez estructural de la plancha, al mismo tiempo que se reduce su masa, por lo que se consigue aumentar su frecuencia natural de vibración, mejorando así la respuesta del sistema, dado que los procesos para los que las nuevas plataformas han sido diseñadas se sitúan en el espectro bajo de frecuencias.

Finalmente, se dispone de circuitería electrónica especializada, alojada en el interior de la plataforma, para amplificar y transmitir los datos a un ordenador, donde 
son almacenados y procesados. La cadena de medida consta, básicamente, de un multiplexor analógico, un amplificador diferencial de precisión, un convertidor analógico-digital de 12 bits y una interfase de comunicaciones conectada a un ordenador procesal, donde reside el software específicamente diseñado en el Instituto para este instrumento. El sistema permite obtener velocidades de muestreo de hasta $1000 \mathrm{~Hz}$ para una plataforma y de hasta $500 \mathrm{~Hz}$ para dos.

La puesta a punto del equipo se realiza en dos etapas. En primer lugar se calibran independientemente los cuatro captadores en condiciones de laboratorio. En segundo lugar, se requiere un ajuste final en el transcurso de su instalación definitiva, siempre a cargo de personal especializado.

Para su funcionamiento correcto se requiere su fijación rígida y estable al suelo. Normalmente se habilita un foso o se construye una pista elevada sobre el suelo. La plataforma, de aproximadamente $9 \mathrm{~cm}$ de altura, queda anclada y a nivel y permite el estudio de marcha humana y movimientos deportivos diversos.

En cierto casos basta con garantizar un apoyo firme del instrumento, como en estudios de equilibrio postural, donde el sujeto permanece bien en reposo sobre la plataforma o bien ejerce acciones horizontales de escasa magnitud.

Se estudia a continuación un ejemplo práctico de aplicación de la plataforma al estudio de la Marcha Humana. Se considera un sujeto que camina sobre ella, apoyando uno de sus pies sobre la placa superior.

La fuerza ejercida por el sujeto sobre la plataforma es absorbida por los cuatro captadores (figura 2). Se general así ocho señales: cuatro correspondientes a fuerzas verticales, dos a antero-posteriores y dos a medio-laterales.

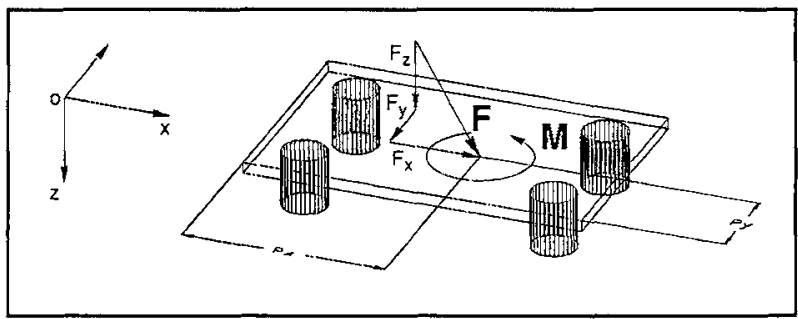

Fig. 2. Distribución de la fuerza de reacción entre los cuatro captadores.

A partir de dichas señales se calculan las tres componentes de la fuerza de reacción, las coordenadas del centro de presión vertical y el momento torsor sobre la plataforma.

El software desarrollo en el Instituto de Biomecánica contempla la representación gráfica de las cargas de reacción frente al tiempo, donde aparece la evolución de las componentes vertical, antero-posterior y medio-lateral de la fuerza.

Se obtienen también los diagramas de los vectores fuerza (figura 3), consistentes en representar la reacción sujeto-suelo, en magnitud y dirección, sobre su punto de aplicación. Igualmente pueden obtenerse representaciones de magnitudes derivadas, como el momento torsor, la velocidad de desplazamiento del punto de aplicación y el impulso mecánico ejercido por el sujeto, entre otras.

En función del proceso de que se trate se calculan, a través del software mencionado, diversos parámetros significativos específicos del mismo. Por ejemplo, para el estudio de la marcha humana, abordado generalmente con dos plataformas gemelas, se obtienen, entre otros, el tiempo de paso, transcurrido entre los apoyos de uno y otro pie, la cadencia, o número de pasos por unidad de tiempo, la longitud del paso, el tiempo de apoyo de cada pie, o intervalo durante el cual un pie se encuentra apoyado sobre el suelo, el tiempo de oscilación de cada pie, o intervalo durante el cual un pie bascula entre dos apoyos consecutivos y el tiempo de doble apoyo, o de apoyo simultáneo de ambos pies.

Asimismo se calculan los valores máximos y mínimos de las tres componentes de la fuerza de reacción y se realiza el análisis armónico de la componente vertical.

Para estudios de estabilidad postural, necesarios en neurología y otorrinolaringología, y que suelen realizarse sobre una única plataforma, se obtiene además la trayectoria del centro de presiones verticales o estabilograma. En este caso se consideran los siguientes parámetros, relativos al centro de presiones:

- Máxima oscilación longitudinal.

- Máxima oscilación transversal.

- Superficie de oscilación.

- Velocidad media de desplazamiento.

- Frecuencia promedio de oscilación.

Las plataformas dinamométricas están integradas en el Laboratorio de Análisis de Movimientos del Instituto de Biomecánica de Valencia, actualmente dotado de electrogoniómetros, de cámaras de cine de alta velocidad y de un analizador de gases. Está prevista su ampliación a corto plazo mediante un sistema de fotogrametría tridimensional de cámaras de vídeo, un podómetro de precisión, plantillas instrumentadas y un sistema de electromiografía.

4 marcha. In 11-22.1988 20:16:36 200 muestras $15 \mathrm{~ms}$. $666 \mathrm{M}$.

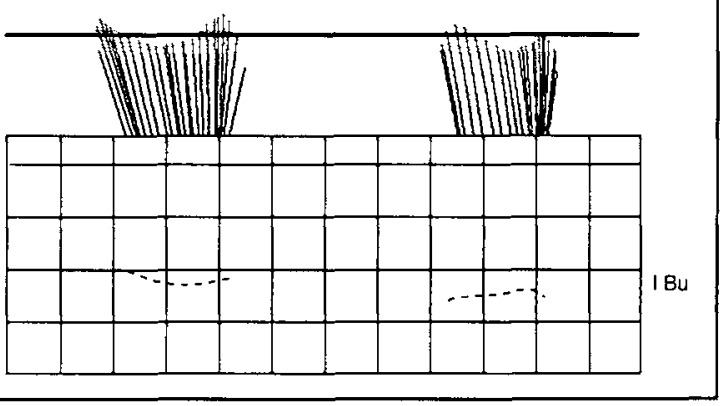

Fig. 3. Diagrama de los vectores fuerza. 